International Journal on Cybernetics \& Informatics (IJCI) Vol. 5, No. 2, April 2016

\title{
CASSANDRA A DISTRIBUTED NOSQL DATABASE FOR Hotel MANAGEMENT SYSTEM
}

\author{
Varalakshmi P. ${ }^{1}$, Hima S. ${ }^{2}$ and Surekha Mariam Varghese ${ }^{3}$ \\ Department of Computer Science and Engineering, M.A. College of Engineering, \\ Kothamangalam, Kerala, India
}

\begin{abstract}
Apache Cassandra is a distributed storage system for managing very large amounts of structured data. Cassandra provides highly available service with no single point of failure. Cassandra aims to run on top of an infrastructure of hundreds of nodes possibly spread across different data centers with small and large components fail continuously. Cassandra manages the persistent state in the face of the failures which drives the reliability and scalability of the software systems. Cassandra does not support a full relational data model because it resembles a database and shares many design and implementation strategies. In this paper, discuss an implementation of Cassandra as Hotel Management System application. Cassandra system was designed to run on cheap commodity hardware. Cassandra provides high write throughput and read efficiency.
\end{abstract}

\section{KEYWORDS}

Cassandra, Data model.

\section{INTRODUCTION}

Apache Cassandra is an open source, distributed, highly available, decentralized, elastically scalable, fault-tolerant, consistent, column-oriented database. Cassandra's distribution design is based on Amazon's Dynamo and its data model on Google's Bigtable. Cassandra was introduced at Facebook; it is now used at some of the most popular sites on the Web [1].

Apache Cassandra is a type of NoSQL database designed to handle large amounts of data across many servers. This database provides high availability and no single point of failure.

Some of the important points of Apache Cassandra: (1) It is scalable, consistent and faulttolerant, (2) It is key-value as well as column-oriented database,(3) Its data model is based on Google's Bigtable and distribution design is based on Amazon's Dynamo, (4) Introduced at Facebook, it differs sharply from relational database management systems,(5) Cassandra implements a Dynamo-style replication model, also adds a more powerful "column family" data model, and (6) Cassandra is being used by some of the biggest companies such as Facebook, Twitter, Cisco, Rackspace, ebay, Twitter, Netflix, and more.

Cassandra has become so popular because of its outstanding technical features. Given below are some of the features of Cassandra:

- Elastic scalability: Cassandra allows adding more hardware to accommodate more customers and more data as per requirement.

- Always on architecture: Cassandra is continuously available for critical business applications that cannot afford single point of failure. 
International Journal on Cybernetics \& Informatics (IJCI) Vol. 5, No. 2, April 2016

- Fast linear-scale performance: Cassandra increases throughput as the number of nodes in the cluster is increased. Therefore it provides a quick response time.

- Flexible data storage: Cassandra handles all possible data formats including: structured, semi-structured, and unstructured. It can dynamically provide changes to data structures according to user need.

- Easy data distribution: Cassandra provides the flexibility to distribute data where user need by replicating data across multiple data centers.

- Transaction support: Cassandra supports properties like Atomicity, Consistency, Isolation, and Durability (ACID).

- Fast writes: Cassandra was designed to run on cheap commodity hardware. It performs fast writes and can store hundreds of terabytes of data, without sacrificing the read efficiency.

The rest of this paper is organized as follows. Section 2 discusses NoSQL database. Section 3 presents the Cassandra Architecture. Section 4 describes the data model of Cassandra. Section 5 describes the implementation details of Hotel Management System. The conclusion is given in Section 6.

\section{NOSQL DATABASE}

A NoSQL database (also called as Not Only SQL) is a database that provides a mechanism to store and retrieve data other than the tabular relations used in relational databases. These databases are schema-free, support easy replication, have simple API, eventually consistent, and can handle huge amounts of data.

The primary objective of a NoSQL database is to have

- simplicity of design,

- horizontal scaling, and

- finer control over availability.

NoSql databases use different data structures compared to relational databases. It makes some operations faster in NoSQL. The suitability of a given NoSQL database depends on the problem it must solve.

\section{CASSANDRA ARCHITECTURE}

The design goal of Cassandra is to handle big data workloads across multiple nodes without any single point of failure. Cassandra has peer-to-peer distributed system, and data is distributed among all the nodes in a cluster [2].

- All the nodes in a cluster play the same role. Each node is independent and at the same time interconnected to other nodes.

- Each node in a cluster can accept read and write requests, regardless of where the data is actually located in the cluster.

- When a node goes down, read/write requests can be served from other nodes in the network. 


\subsection{Data Replication In Cassandra}

In Cassandra, one or more of the nodes in a cluster act as replicas for a given piece of data. If it is detected that some of the nodes responded with an out-of-date value, Cassandra will return the most recent value to the client. After returning the most recent value, Cassandra performs a read repair in the background to update the stale values.

The figure 1 shows a schematic view of how Cassandra uses data replication among the nodes in a cluster to ensure no single point of failure. Cassandra uses the Gossip Protocol to allow the nodes to communicate with each other and detect any faulty nodes in the cluster.

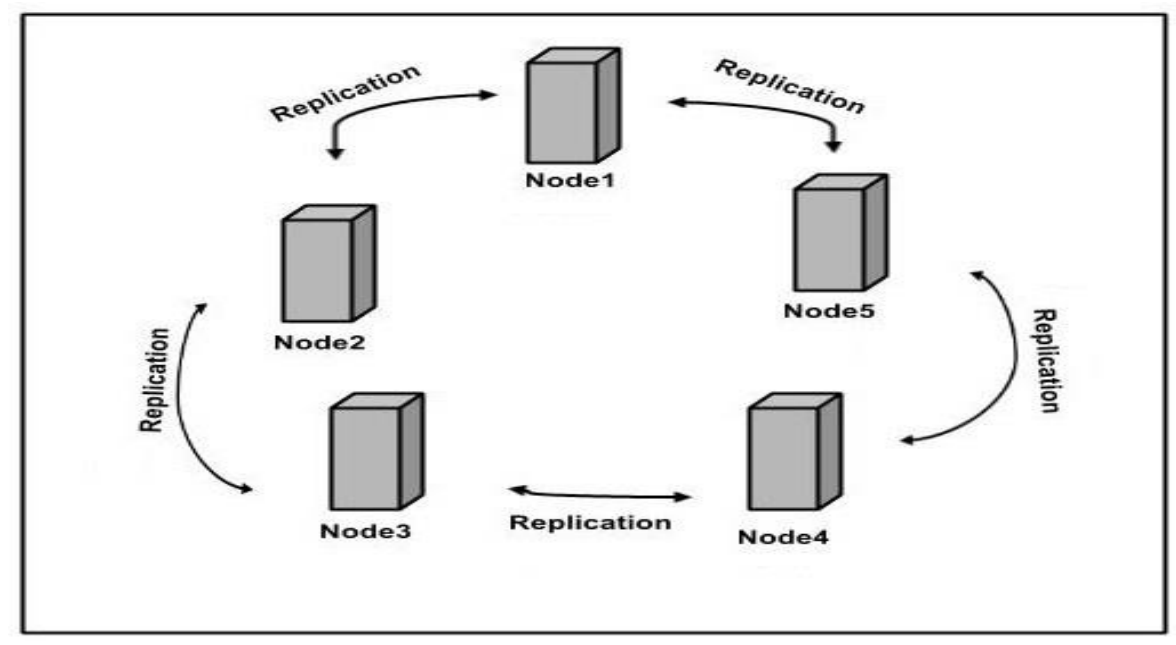

Figure. 1 Schematic view of Cassandra

\subsection{Components of Cassandra}

The key components of Cassandra are as follows:

- Node: It is the place where data is stored.

- Data center: It is a collection of related nodes.

- Cluster: A cluster is a component that contains one or more data centers.

- Commit log: The commit log is a crash-recovery mechanism in Cassandra. Every write operation is written to the commit log.

- Mem-table: A mem-table is a memory-resident data structure. After commit log, the data will be written to the mem-table. Sometimes, for a single-column family, there will be multiple mem-tables.

- SSTable: It is a disk file to which the data is flushed from the mem-table when its contents reach a threshold value.

- Bloom filter: These are quick, nondeterministic, algorithms for testing whether an element is a member of a set. It is a special kind of cache. Bloom filters are accessed after every query. 
International Journal on Cybernetics \& Informatics (IJCI) Vol. 5, No. 2, April 2016

\subsection{Cassandra Query Language}

Users can access Cassandra through its nodes using Cassandra Query Language (CQL). CQL treats the database (Keyspace) as a container of tables. Programmers use cqlsh: a prompt to work with CQL or separate application language drivers.

\subsection{Write Operations}

Every write activity of nodes is captured by the commit logs written in the nodes. Then the data will be captured and stored in the mem-table. Whenever the mem-table is full, data will be written into the SStable data file. All writes are automatically partitioned and replicated throughout the cluster. Cassandra periodically consolidates the SSTables, deleting unnecessary data.

\subsection{Read Operations}

During read operations, Cassandra gets values from the mem-table. It checks the bloom filter to find the appropriate SSTable that holds the required data.

\section{DATA MODEL}

The data model of Cassandra is significantly different from the normal RDBMS [2].

\subsection{Cluster}

Cassandra database is distributed over several machines that operate together [3]. The outermost container is known as the Cluster. For failure handling, every node contains a replica. In case of a failure, the replica takes charge. Cassandra arranges the nodes in a cluster, in a ring manner, and assigns data to them.

\subsection{Keyspace}

Keyspace is the outermost container for data in Cassandra. The basic attributes of a Keyspace in Cassandra are:

- Replication factor: It is the number of machines in the cluster that will receive copies of the same data.

- Replica placement strategy: It is the strategy to place replicas in the ring. The different strategies such as simple strategy (rack-aware strategy), old network topology strategy (rack-aware strategy), and network topology strategy (data center-shared strategy) are available.

- Column families: Keyspace is a container for a list of one or more column families. A column family is a container of a collection of rows. Each row contains ordered columns. Column families represent the structure of data. Each keyspace has at least one and often many column families. 


\section{IMPLEMENTATION DETAILS}

The implementation of Apache Cassandra includes installing and configuring Cassandra. Initially download Cassandra from cassandra.apache.org. Copy the folder named cassandra. Move to bin folder. Open the Cassandra.yaml file which is available in the bin folder of the Cassandra folder. Verify that the following configurations.

- data_file_directories"/var/lib/cassandra/data"

- commitlog_directory “/var/lib/cassandra/commitlog"

- saved_caches_directory“/var/lib/cassandra/saved_caches"

\section{Setting the path}

Set the path as Cassandra_Home=C:Iapache-cassandra-1.2.19

Starting Cassandra

\$ cd \$CASSANDRA_HOME

\$./bin/cassandra $-\mathrm{f}$

\section{Starting cqlsh}

Start cqlsh using the command cqlshas shown below. It gives the Cassandra cqlsh prompt as output.

\$ cqlsh Connected to Test Cluster at 127.0.0.1:9042.

[cqlsh 5.0.1 | Cassandra 2.1.2 | CQL spec 3.2.0 | Native protocol v3]

cqlsh>

An application of Cassandra implementation is Hotel Management System (HMS) [5].Cassandra database is chosen for this application because of its increasing throughput as the number of nodes increases, continuous availability for critical business applications and elastic scalability. Moreover Cassandra handles all possible data formats and distribution of data by replicating data across multiple data centres. Cassandra supports ACID properties and it works on cheap commodity hardware.

In the keyspace of Hotel Management System Figure 2 we have the followingcolumn families: Hotel, HotelByCity, Guest, Reservation, PointOfInterest, Room, Room Availability.

In this design, transferred some of the tables, such as Hotel and Guest, to column families. Other tables, such as PointOfInterest, have been denormalized into a super column family. We have created an index in the form of the HotelByCity column family.

We have combined room and amenities into a single column family, Room. The columns such as type and rate will have corresponding values; other columns, such as hot tub, will just use the presence of the column name itself as the value, and be otherwise empty.

Hotel Management System includes details about different hotels, guests who stay in the hotels, availability of rooms for each hotel, and a record of the reservation, which is a certain guest in a certain room for a certain period of time (called the "stay"). Hotels typically also maintain a collection of "points of interest," which are shopping galleries, monuments, museums, parks, or other places near the hotel that guests might like to visit during their stay.

Our application Hotel Management System designed with Cassandra includes the following characteristics: 
International Journal on Cybernetics \& Informatics (IJCI) Vol. 5, No. 2, April 2016

- Find hotels in a given area.

- Find information about a specific hotel, such as its name, location, room availability etc.

- Find interesting locations near to a given hotel.

- Find availability of rooms in a given date range.

- Find the amenities and rate for a room.

- Possible to book the selected rooms by entering guest information.

The database in Cassandra is created using keyspace. A keyspace in Cassandra is a namespace which defines data replication on nodes. A cluster contains one keyspace per node.

The application we're building will do the following things:

1. Create the database structure.

2. Prepopulate the database with hotel and point of interest data. The hotels are stored in standard column families, and the points of interest are in super column families.

3. Search for a list of hotels in a given city. This uses a secondary index.

4. Select one of the hotels returned in the search, and then search for a list of points of interest near the chosen hotel.

5. Booking the hotel by doing an insert into the Reservation column family should be straightforward at this point, and is left to the reader.

\begin{tabular}{|c|c|c|}
\hline$\ll$ CF $\gg$ Hotel & $<<$ CF $\gg$ HotelByCity & $\ll S C F \gg$ PointOfinterest \\
\hline \multirow{3}{*}{$\begin{array}{l}\text { «RowKey } \gg \text { \#hotelID } \\
\text { + name } \\
\text { +phone } \\
\text { + address } \\
\text { +city } \\
\text { + state } \\
\text { +zip }\end{array}$} & $\begin{array}{l}\ll \text { RowKey } \gg \text { \#city:state:hotellD } \\
+ \text { hotel1 } \\
\text { +hotel2 } \\
+\ldots\end{array}$ & $\begin{array}{l}\ll \text { SuperColumnName } \gg \text { \#hotelID } \\
\ll<\text { RowKey } \gg \text { \#poiName } \\
\text { +desc } \\
\text { +Phone }\end{array}$ \\
\hline & & \\
\hline & $\ll$ SCF $\gg$ RoomAvailablity & $\ll$ SCF $\gg$ Room \\
\hline \multirow{2}{*}{$\ll \mathrm{CF} \gg$ Guest } & $\begin{array}{l}\ll \text { SuperColumnName } \gg \text { \#hotellD } \\
\ll \text { RowKey } \gg+\text { date }\end{array}$ & \multirow{6}{*}{$\begin{array}{l}\ll \text { SuperColumnName } \gg \text { \#hotelID } \\
\ll \text { RowKey } \gg \text { \#roomID } \\
\text { + num } \\
\text { +type } \\
\text { +rate } \\
\text { + coffee } \\
\text { +tv } \\
\text { +hottub } \\
\text { +... }\end{array}$} \\
\hline & $+\mathbf{k k}:<$ unspecified $>=22$ & \\
\hline \multirow{5}{*}{$\begin{array}{l}\ll \text { RowKey } \gg \text { \#phone } \\
+ \text { fname } \\
+ \text { +Iname } \\
\text { +email }\end{array}$} & $+\mathrm{qq}:<$ unspecified $>=14$ & \\
\hline & & \\
\hline & $\ll<F \gg$ Reservation & \\
\hline & \multirow[b]{2}{*}{$\begin{array}{l}\text { «RowKey } \gg \text { \#resID } \\
\text { + hotellD } \\
\text { +roomID } \\
\text { + phone } \\
\text { + name } \\
\text { + arrive } \\
\text { +depart } \\
\text { +rate } \\
\text { +ccNum }\end{array}$} & \\
\hline & & \\
\hline & & \\
\hline
\end{tabular}

Figure. 2 Hotel Management System

\subsection{Table Operations}

To create a table use the command CREATE TABLE. The tables required for the Hotel Management System application can be created using this command. The syntax is CREATE (TABLE | COLUMNFAMILY) <tablename> ('<column-definition>', '<columndefinition>') 
International Journal on Cybernetics \& Informatics (IJCI) Vol. 5, No. 2, April 2016

The primary key is represented by a column that is used to uniquely identify a row. Therefore, defining a primary key is mandatory while creating a table. A primary key is also made of one or more columns of a table [4].

\subsection{CURD Operations}

To create data in a table use the command INSERT. The syntax for creating data in a table is INSERT INTO <tablename> (<column1 name>, <column2 name>....) VALUES (<value1>, <value2>....)

UPDATE is the command used to update data in a table. The syntax of update is

UPDATE <tablename > SET < column name $>=\langle$ new value $>$

$<$ column name $>=\langle$ value $>$... WHERE $<$ condition $>$

Reading Data using SELECT Clause from a table in Cassandra. Using this clause we can read a whole table, a single column, or a particular cell. The syntax of SELECT is

SELECT FROM < table name> WHERE < condition>

Delete data from a table using the command DELETE. Its syntax is

DELETE FROM <identifier> WHERE <condition>

\subsection{Performance Evaluation}

One of the hallmarks of Cassandra is its high performance, for both reads and writes operations. When new nodes are added to a cluster, Cassandra scales it linearly. The performance of Hotel Management System application is evaluated with various hardware requirements such as Intel core CPU @ $1.80 \mathrm{GHz}, 64$-bit operating system, x64 based processor, 4.00GB RAM. The software specifications include Apache Cassandra version 1.2.19. Figure 3 gives performance of Cassandra operations.

In the graph of performance evaluation of Cassandra database $X$ axis represents the throughput in ops/sec and $\mathrm{Y}$ axis represents average latency in ms. Here three operations such as update, insert and read are evaluated for performance. In the graph it is clear that update operation has very high throughput while it is in low latency. Similarly insert operation has high throughput [6] while it is in low latency which is greater than latency of update operation. In the case of read operation which has low throughput while it is in high latency.

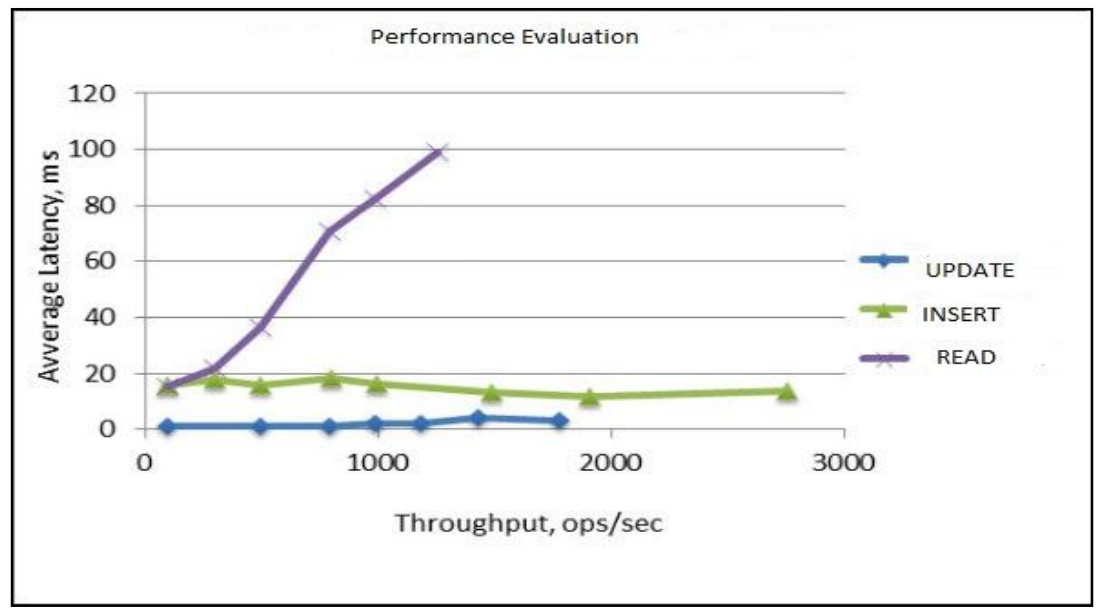

Figure.3.Performance Evaluation 


\section{CONCLUSION}

NoSQL database: Cassandra is built, implemented, and operated a scalable storage system providing high performance, and wide applicability. Demonstrated that Cassandra can support a very high update throughput while delivering low latency. It is very efficient as compared with other databases.

\section{REFERENCES}

[1] http://cassandra.apache.org

[2] http://www.tutorialspoint.com/cassandra/

[3] Dietrich Featherston, Cassandra: Principles and Application, 2010

[4] A. Lakshman, P. Malik, Cassandra - A Decentralized Structured Storage System, Cornell, 2009.

[5] https://www.safaribooksonline.com/library/view/cassandra-thedefinitive/9781449399764/ch04.html

[6] Matthias Nicola and Matthias Jarke. Performance modeling of distributed and replicated databases.IEEE Trans. on Knowl.and Data Eng.,12(4):645-672, July 2000.

\section{Authors}

Varalakshmi P. is currently pursuing M.Tech in Computer Science and Engineering in Mar Athanasius College of Engineering. She completed her B.Tech from P.R.S. College of Engineering and Technology, Thiruvananthapuram. Her areas of research are Data Mining, Databases and Image Processing.

Hima S. is currently pursuing M.Tech in Computer Science and Engineering in Mar Athanasius College of Engineering. She completed her B.Tech from Mohandas College of Engineering and Technology, Thiruvananthapuram. Her areas of research are Image Processing, Database and Data Mining.

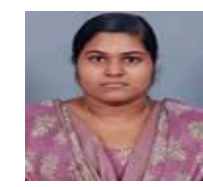

Surekha Mariam Varghese is currently heading the Department of Computer Science and Engineering, M.A. College of Engineering, Kothamangalam, Kerala, India. She received her B-Tech Degree in Computer Science and Engineering in 1990 from College of Engineering, Trivandrum affiliated to Kerala University and M-Tech in Computer and Information Sciences from Cochin University of Science and Technology, Kochi in 1996. She obtained Ph.D in Computer Security from Cochin University of Science and

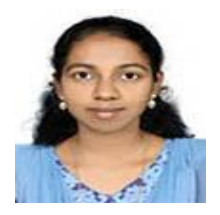
Technology, Kochi in 2009. She has around 25 years of teaching and research experience in various institutions in India. Her research interests include Network Security, Database Management, Data Structures and Algorithms, Operating Systems and Distributed Computing, Machine learning. She has published 17 papers in international journals and international conference proceedings. She has been in the chair for many international conferences and journals. 